\title{
REGULARITY OF SOLUTIONS OF TWO-DIMENSIONAL MONGE-AMPĖRE EQUATIONS
}

\author{
FRIEDMAR SCHULZ AND LIANG-YUAN LIAO
}

\begin{abstract}
In the paper we investigate the regularity of solutions $z(x, y) \epsilon$ $C^{1,1}(\Omega)$, resp. $C^{1,1}(\bar{\Omega})$ of elliptic Monge-Ampère equations of the form

$$
A r+2 B s+C t+\left(r t-s^{2}\right)=E .
$$

It is shown that $z(x, y) \in C^{2, \alpha}(\Omega)$, resp. $C^{2, \alpha}(\bar{\Omega})$, with corresponding a priori estimates, if $A, B, C, E \in C^{\alpha}\left(\Omega \times \mathbf{R}^{3}\right)$. The results are deduced via the Campanato technique for equations of variational structure invoking a Legendre-like transformation.
\end{abstract}

I. Introduction and statement of the result. Let $\Omega$ be an open subset of the $x, y$-plane. We shall consider solutions $z(x, y)$ of class $C^{1,1}(\Omega)$ of elliptic Monge-Ampère equations of the general form

$$
A r+2 B s+C t+\left(r t-s^{2}\right)=E \quad\left(\Delta=A C-B^{2}+E>0\right) .
$$

The coefficients $A, B, C, E$ are assumed to belong to the Hölder class $C^{\alpha}(0<\alpha<1)$ with respect to the variables $x, y, z, p, q$. Here $p, q, r, s, t$ represent the first and second derivatives of $z(x, y)$.

Suppose $a$ is a bound for the absolute values of $A, B, C, E$, and suppose $b$ is a bound for their Hölder constants. Furthermore let $1 / c$ be a lower bound for $\Delta$ and $K$ be a bound for the $C^{1,1}$-norm of $z(x, y)$. The first result of the paper then reads as the following

THEOREM 1. The second derivatives of $z(x, y)$ are Hölder continuous in $\Omega$ with exponent $\alpha$. In every subset $\Omega^{\prime}$, which is compactly contained in $\Omega$, they satisfy the Hölder conditions

$$
\begin{gathered}
\left|r\left(x^{\prime}, y^{\prime}\right)-r\left(x^{\prime \prime}, y^{\prime \prime}\right)\right|, \ldots,\left|t\left(x^{\prime}, y^{\prime}\right)-t\left(x^{\prime \prime}, y^{\prime \prime}\right)\right| \\
\leq H\left(\left(x^{\prime}-x^{\prime \prime}\right)^{2}+\left(y^{\prime}-y^{\prime \prime}\right)^{2}\right)^{\alpha / 2}
\end{gathered}
$$

$\left(\left(x^{\prime}, y^{\prime}\right),\left(x^{\prime \prime}, y^{\prime \prime}\right) \in \Omega^{\prime}\right)$, where the constant $H$ only depends on $\alpha, a, b, c, K$ and the distance between $\Omega^{\prime}$ and $\partial \Omega$.

Suppose now $\Omega$ is a bounded open set with boundary $\partial \Omega$ of class $C^{2, \alpha}$ and let $\varphi \in C^{2, \alpha}(\partial \Omega)$, with $k$ being a bound for its $C^{2, \alpha}(\partial \Omega)$-norm. The second part of the paper is concerned with the regularity near $\partial \Omega$ of solutions $z(x, y) \in C^{1,1}(\bar{\Omega})$ of the Dirichlet problem for the equation (1) subject to the boundary condition

Received by the editors August 3, 1986 and, in revised form, March 26, 1987. The contents of this paper have been presented to the American Mathematical Society at the 93rd Annual Meeting in San Antonio, Texas, January 21-24, 1987.

1980 Mathematics Subject Classification (1985 Revision). Primary 35B45, 35D10, 35J60; Secondary $35 \mathrm{~A} 30$.

The first author was supported in part by grant DMS-8603619 from the National Science Foundation. 
$\left.z\right|_{\partial \Omega}=\varphi$. The result can be stated as

THEOREM 2. The second derivatives of $z(x, y)$ are Hölder continuous in $\bar{\Omega}$ with exponent $\alpha$. They satisfy the Hölder conditions (2) for $\left(x^{\prime}, y^{\prime}\right),\left(x^{\prime \prime}, y^{\prime \prime}\right) \in \Omega$, where the constant $H$ depends only on $\alpha, a, b, c, k, K$ and $\Omega$.

The regularity parts of the theorems complete the results obtained by the author in $[\mathbf{1 2}-\mathbf{1 5}]$. The also stated interior a priori estimates have been established in $[\mathbf{1 4}]$, the boundary estimates in [15], if $u(x)$ is known to be regular, i.e., of class $C^{2, \alpha}(\Omega)$, resp. $C^{2, \alpha}(\bar{\Omega})$. The above-cited papers are subsequent to massive classical work, in particular by H. Lewy [7], A. D. Aleksandrov [1], Bakel'man [2], Pogorelov [9], Nirenberg [8], Heinz [6] and Sabitov [10]. We refer the reader to the account given in $[\mathbf{1 4}]$.

In addition, it should be noted that Trudinger [17] has recently shown a regularity result for fully nonlinear, concave equations in $n$ dimensions under certain differentiabilty assumptions. The class of equations under consideration includes general Monge-Ampère equations of the form (1). Such results can also be obtained via the Green's function technique, as mentioned in [16], regularity results actually somewhat easier than the therein proven a priori estimates. We also wish to draw attention to Safonov's approach [11], which yields the existence of an $\bar{\alpha}, 0<\bar{\alpha}<1$, such that the estimates of Theorems 1 and 2 hold for $\alpha<\bar{\alpha}$, if the coefficients belong to $C^{\bar{\alpha}}$. The purpose of the present paper is to cover the case of merely $\alpha$-Hölder-continuous coefficients $(0<\alpha<1)$.

Like [14], the proof of Theorem 1 is based on a Legendre-like transformation. But then the proof rests on a technique due to Campanato [3, 4], unlike [14], where the Schauder technique was employed, which unavoidably requires the regularity $z(x, y) \in C^{2, \alpha}(\Omega)$. At the beginning of the second section we shall review from $[\mathbf{1 4}]$, incorporating the necessary changes.

Similar remarks apply to the proof of Theorem 2. Like [15], we first straighten $\partial \Omega$ locally. It is then important to introduce the zero-boundary data not too early, i.e., only after performing the transformation.

It should also be noted, that we have achieved a few simplifications over [14, 15]. We adopt the notation used therein. The letter $C$ denotes various constants, which may change from line to line. If possible, we choose constants to be $\geq 1$.

II. Proof of Theorem 1. Suppose $D_{R}=D_{R}\left(x_{0}, y_{0}\right)$ is a circular disc of radius $R>0$, centered at $\left(x_{0}, y_{0}\right) \in \Omega$. The very first thing to note is that the equation (1) can be written in the equivalent form

$$
(r+C)(t+A)-(s-B)^{2}=\Delta .
$$

By putting

$$
A_{0}:=A\left(x_{0}, y_{0}, \ldots, q\left(x_{0}, y_{0}\right)\right), \quad \ldots, \quad E_{0}:=E(\cdots), \quad \Delta_{0}:=\Delta(\cdots),
$$

the equation (1) can therefore be rewritten in the form

$$
\begin{aligned}
& \left(r+C_{0}\right)\left(t+A_{0}\right)-\left(s-B_{0}\right)^{2} \\
& \quad=\Delta_{0}+\left(\left(A_{0}-A\right) r+2\left(B_{0}-B\right) s+\left(C_{0}-C\right) t+\left(E-E_{0}\right)\right) \\
& \quad=: \tilde{f}(x, y) .
\end{aligned}
$$


Hence the function

$$
\begin{aligned}
\tilde{z}(x, y):= & z(x, y)+\frac{1}{2}\left(C_{0}\left(x-x_{0}\right)^{2}-2 B_{0}\left(x-x_{0}\right)\left(y-y_{0}\right)+A_{0}\left(y-y_{0}\right)^{2}\right) \\
& +2(K+a)\left(y-y_{0}\right)
\end{aligned}
$$

solves the equation

$$
\tilde{r} \tilde{t}-\tilde{s}^{2}=\tilde{f}(x, y) \geq 1 / 2 c=: 1 / \tilde{c}>0
$$

in $D:=D_{R_{0}}\left(x_{0}, y_{0}\right)$, where

$$
R_{0}:=\min \{1 / \sqrt[\alpha]{10 b c K}, d / 2\} .
$$

Here $d>0$ is a lower bound for the distance between $\left(x_{0}, y_{0}\right)$ and $\partial \Omega$. We furthermore have the estimates $\tilde{q} \geq 1,|\tilde{r}|,|\tilde{t}| \geq 1 / \tilde{c} \tilde{K}$, where $\tilde{K} \geq 1$ is a bound for the $C^{1,1}$-norm of $\tilde{z}(x, y)$.

Now we make the variable transformation

$$
T:\left\{\begin{array}{l}
u=x \\
v=\tilde{q}(x, y)
\end{array}\right.
$$

$((x, y) \in D)$. Compare also [15, Lemma 3], for the following list of its properties:

(i) $T$ maps $D$ homeomorphically onto the image $T(D)$.

(ii) For $\left(x^{\prime}, y^{\prime}\right),\left(x^{\prime \prime}, y^{\prime \prime}\right) \in D$, we have the dilation estimates

$$
\begin{aligned}
& \left(u^{\prime}-u^{\prime \prime}\right)^{2}+\left(v^{\prime}-v^{\prime \prime}\right)^{2} \leq \gamma_{1}^{2}\left(\left(x^{\prime}-x^{\prime \prime}\right)^{2}+\left(y^{\prime}-y^{\prime \prime}\right)^{2}\right), \\
& \left(x^{\prime}-x^{\prime \prime}\right)^{2}+\left(y^{\prime}-y^{\prime \prime}\right)^{2} \leq \gamma_{2}^{2}\left(\left(u^{\prime}-u^{\prime \prime}\right)^{2}+\left(v^{\prime}-v^{\prime \prime}\right)^{2}\right),
\end{aligned}
$$

with constants $\gamma_{1}, \gamma_{2} \geq 1$, depending only on $\tilde{c}, \tilde{K}$.

(iii) Hence the inclusions

$$
T\left(D_{R / \gamma_{1}}\left(x_{0}, y_{0}\right)\right) \subset D_{R}\left(u_{0}, v_{0}\right), \quad D_{R / \gamma_{2}}\left(u_{0}, v_{0}\right) \subset T\left(D_{R}\left(x_{0}, y_{0}\right)\right)
$$

hold for all $R, 0<R \leq R_{0}$.

(iv) The function $y(u, v) \in C^{0,1}(T(D))$ is a weak solution of the equation

$$
y_{u u}+\left(\tilde{f} y_{v}\right)_{v}=0 \text {. }
$$

Only the proof of (4) needs to be modified slightly: On estimating

$$
\begin{aligned}
\left|v^{\prime}-v^{\prime \prime}\right| & =\left|\int_{0}^{1}\left\{s\left((1-\tau) x^{\prime}+\tau x^{\prime \prime},(1-\tau) y^{\prime}+\tau y^{\prime \prime}\right)\left(x^{\prime}-x^{\prime \prime}\right)+t(\cdots)\left(y^{\prime}-y^{\prime \prime}\right)\right\} d \tau\right| \\
& \geq-\tilde{K}\left|x^{\prime}-x^{\prime \prime}\right|+\frac{1}{\tilde{c} \tilde{K}}\left|y^{\prime}-y^{\prime \prime}\right|,
\end{aligned}
$$

we obtain

$$
\begin{aligned}
\left(u^{\prime}-\right. & \left.u^{\prime \prime}\right)^{2}+\left(v^{\prime}-v^{\prime \prime}\right)^{2} \\
& \geq\left(1+\tilde{K}^{2}\right)\left(x^{\prime}-x^{\prime \prime}\right)^{2}-\frac{2}{\tilde{c}}\left|x^{\prime}-x^{\prime \prime}\right|\left|y^{\prime}-y^{\prime \prime}\right|+\frac{1}{(\tilde{c} \tilde{K})^{2}}\left(y^{\prime}-y^{\prime \prime}\right)^{2} \\
& \geq\left(1+\left(1-\frac{1}{\delta}\right) \tilde{K}^{2}\right)\left(x^{\prime}-x^{\prime \prime}\right)^{2}+\frac{1-\delta}{(\tilde{c} \tilde{K})^{2}}\left(y^{\prime}-y^{\prime \prime}\right)^{2} .
\end{aligned}
$$

Inequality (4) then follows by choosing $\delta$ to be the mean of $\tilde{K}^{2} /\left(1+\tilde{K}^{2}\right)$ and 1 . 
Noting that

$$
\left(\begin{array}{ll}
x_{u} & x_{v} \\
y_{u} & y_{v}
\end{array}\right)=\left(\begin{array}{cc}
1 & 0 \\
-\tilde{s} / \tilde{t} & 1 / \tilde{t}
\end{array}\right)
$$

we proceed to rewrite equation (5) in the form

$$
y_{u u}+\left(\Delta_{0} y_{v}\right)_{v}=g_{v}
$$

where

$$
g(u, v):=\left(\left(A-A_{0}\right) r+2\left(B-B_{0}\right) s-\left(C-C_{0}\right) t+\left(E_{0}-E\right)\right) / \tilde{t} .
$$

We can now apply the Campanato technique $[3,4]$ as described in Chapter III of Giaquinta's book [4]. It is unnecessary to carry out the first step, [5, Theorem 3.1 , because of the boundedness of $\nabla y(u, v)$. As in the proof of [5, Theorem 3.2, resp. Theorem 2.2], we obtain the inequalities

$$
\begin{aligned}
& \iint_{D_{\rho}}\left|\nabla y-(\nabla y)_{\rho}\right|^{2} d u d v \\
& \quad \leq C\left\{\left(\frac{\rho}{R}\right)^{4} \iint_{D_{R}}\left|\nabla y-(\nabla y)_{R}\right|^{2}+\iint_{D_{R}}\left|g-g_{R}\right|^{2}\right\}
\end{aligned}
$$

for all $\rho \leq R \leq R_{0} / \gamma_{2}$. Here all discs are centered at $\left(u_{0}, v_{0}\right)$ and

$$
g_{R}:=\frac{1}{\left|D_{R}\right|} \iint_{D_{R}} g(u, v) d u d v \text {. }
$$

Using the dilation inequality (3), we estimate

$$
\iint_{D_{R}}\left|g-g_{R}\right|^{2} \leq C \iint_{D_{R}}|g|^{2} \leq C R^{2+2 \alpha},
$$

where the last constant also depends on $b$. It therefore follows that

$$
\iint_{D_{\rho}}\left|\nabla y-(\nabla y)_{\rho}\right|^{2} d u d v \leq C\left\{\left(\frac{\rho}{R}\right)^{4} \iint_{D_{R}}\left|\nabla y-(\nabla y)_{R}\right|^{2}+R^{2+2 \alpha}\right\} .
$$

An iteration argument, [5, Lemma 2.1], yields for all $\rho \leq R \leq R_{0} / \gamma_{2}$

$$
\begin{aligned}
\iint_{D_{\rho}}\left|\nabla y-(\nabla y)_{\rho}\right|^{2} d u d v & \leq C\left\{\left(\frac{\rho}{R}\right)^{2+2 \alpha} \iint_{D_{R}}\left|\nabla y-(\nabla y)_{R}\right|^{2}+\rho^{2+2 \alpha}\right\} \\
& \leq \frac{C}{R^{2 \alpha}} \rho^{2+2 \alpha},
\end{aligned}
$$

incorporating the boundedness of $\nabla \eta$.

We are not done, because the variables $u, v$, and therefore also the function $y(u, v)$, depend on the point $\left(u_{0}, v_{0}\right)$. Hence we reintroduce the $x, y$-variables, using some elementary geometric measure theory. By (6), we have a.e.

$$
y_{v}-\left(y_{v}\right)_{\rho}=\frac{1}{\tilde{t}} \frac{\left|T^{-1}\left(D_{\rho}\right)\right|}{\left|D_{\rho}\right|}\left(t_{; \rho}-t\right)
$$

where

$$
g_{; \rho}=g_{\left(x_{0}, y_{0}\right) ; \rho}:=\frac{1}{\left|T^{-1}\left(D_{\rho}\right)\right|} \iint_{T^{-1}\left(D_{\rho}\right)} g(x, y) d x d y
$$


and consequently

$$
\iint_{D_{\rho}}\left|y_{v}-\left(y_{v}\right)_{\rho}\right|^{2} d u d v=\frac{\left|T^{-1}\left(D_{\rho}\right)\right|}{\left|D_{\rho}\right|} \iint_{T^{-1}\left(D_{\rho}\right)} \frac{1}{\tilde{t}}\left|t-t_{; \rho}\right|^{2} d x d y .
$$

The inequalities

$$
\iint_{D_{\rho / \gamma_{1}}\left(x_{0}, y_{0}\right)}\left|t-t_{; \rho}\right|^{2} d x d y \leq \frac{C}{R_{0}^{2 \alpha}} \rho^{2+2 \alpha}
$$

hold therefore for all $\left(x_{0}, y_{0}\right) \in \Omega$ and all $\rho, 0<\rho \leq R_{0} / \gamma_{2}$. Here $t_{; \rho}$ depends on $\left(x_{0}, y_{0}\right)$ through the transformation $T$.

Similarly

$$
y_{u}-\left(y_{u}\right)_{\rho}=\frac{1}{\tilde{t}} \frac{\left|T^{-1}\left(D_{\rho}\right)\right|}{\left|D_{\rho}\right|}\left(\tilde{s}_{; \rho} \tilde{t}-\tilde{s} \tilde{t}_{; \rho}\right)
$$

a.e., hence

$$
\iint_{D_{\rho}}\left|y_{u}-\left(y_{u}\right)_{\rho}\right|^{2} d u d v=\frac{\left|T^{-1}\left(D_{\rho}\right)\right|}{\left|D_{\rho}\right|} \iint_{T^{-1}\left(D_{\rho}\right)} \frac{1}{\tilde{t}}\left|\tilde{s} \tilde{t}_{; \rho}-\tilde{s}_{; \rho} \tilde{t}\right|^{2} d x d y .
$$

On estimating

$$
\left|s-s_{; \rho}\right| \leq\left|\tilde{s}-\tilde{s}_{; \rho} \frac{1}{\tilde{t}_{; \rho}}\right|+\left|\tilde{s}_{; \rho} \frac{1}{\tilde{t}_{; \rho}}-\tilde{s}_{; \rho}\right| \leq C\left\{\left|\tilde{s} \tilde{t}_{; \rho}-\tilde{s}_{; \rho} \tilde{t}\right|+\left|\tilde{t}-\tilde{t}_{; \rho}\right|\right\}
$$

a.e. and using the differential equation (1), we therefore conclude that for all $\left(x_{0}, y_{0}\right) \in \Omega$ and all $\rho, 0<\rho \leq R_{0} / \gamma_{2}$,

$$
\iint_{D_{\rho / \gamma_{1}}\left(x_{0}, y_{0}\right)}\left\{\left|r-r_{; \rho}\right|^{2}+\cdots+\left|t-t_{; \rho}\right|^{2}\right\} d x d y \leq \frac{C}{R_{0}^{2 \alpha}} \rho^{2+2 \alpha} .
$$

We finally deduce the Hölder continuity of $r, s, t$ by proceeding as in the proof of [5, Theorem 1.2], that the Campanato spaces $\mathcal{L}^{2, n+2 \alpha}(\Omega)$ are contained in the Hölder spaces $C^{\alpha}(\Omega)$ for $0<\alpha<1$ (here $n=2$ ). First we get the analogue of formula (1.8) of $[5$, p. 71], that there exist the limits

$$
\bar{r}\left(x_{0}, y_{0}\right):=\lim _{\rho \rightarrow 0} r_{; \rho}, \ldots, \bar{t}\left(x_{0}, y_{0}\right):=\lim _{\rho \rightarrow 0} t_{; \rho}
$$

and

$$
\left|r_{; \rho}-\bar{r}\left(x_{0}, y_{0}\right)\right|, \ldots,\left|t_{; \rho}-\bar{t}\left(x_{0}, y_{0}\right)\right| \leq C \rho^{\alpha}
$$

for $\rho \leq R_{0} / \gamma_{2}$. Here $C$ depends also on $R_{0}$.

Now suppose $\Omega^{\prime}$ is a disc in $\Omega$ with sufficiently small radius, indeed $\Omega^{\prime}:=$ $D_{R_{0} / 2 \gamma_{2}}\left(x_{0}, y_{0}\right)$ would be fine. Let $\left(x^{\prime}, y^{\prime}\right),\left(x^{\prime \prime}, y^{\prime \prime}\right) \in \Omega^{\prime}$ and $\left(\rho / 2 \gamma_{1}\right)^{2}:=$ $\left(x^{\prime}-x^{\prime \prime}\right)^{2}+\left(y^{\prime}-y^{\prime \prime}\right)^{2}$. Then

$$
\begin{aligned}
\left|\bar{t}\left(x^{\prime}, y^{\prime}\right)-\bar{t}\left(x^{\prime \prime}, y^{\prime \prime}\right)\right| \leq & \left|\bar{t}\left(x^{\prime}, y^{\prime}\right)-t_{\left(x^{\prime}, y^{\prime}\right) ; \rho}\right| \\
& +\left|t_{\left(x^{\prime}, y^{\prime}\right) ; \rho}-t_{\left(x^{\prime \prime}, y^{\prime \prime}\right) ; \rho}\right|+\left|t_{\left(x^{\prime \prime}, y^{\prime \prime}\right) ; \rho}-\bar{t}\left(x^{\prime \prime}, y^{\prime \prime}\right)\right| \\
\leq & C \rho^{\alpha}+\left|t_{\left(x^{\prime}, y^{\prime}\right) ; \rho}-t(x, y)\right|+\left|t(x, y)-t_{\left(x^{\prime \prime}, y^{\prime \prime}\right) ; \rho}\right|
\end{aligned}
$$


and by integrating with respect to $(x, y)$ over $D_{\rho / \gamma_{1}}\left(x^{\prime}, y^{\prime}\right) \cap D_{\rho / \gamma_{1}}\left(x^{\prime \prime}, y^{\prime \prime}\right)$, which includes $D_{\rho / 2 \gamma_{1}}\left(x^{\prime}, y^{\prime}\right)$, we thus obtain

$$
\begin{aligned}
\left|\bar{t}\left(x^{\prime}, y^{\prime}\right)-\bar{t}\left(x^{\prime \prime}, y^{\prime \prime}\right)\right| \leq & C \rho^{\alpha}+\frac{1}{\left|D_{\rho / 2 \gamma_{1}}\left(x^{\prime}, y^{\prime}\right)\right|} \\
& \quad \times\left\{\iint_{D_{\rho / \gamma_{1}}\left(x^{\prime}, y^{\prime}\right)}\left|t-t_{\left(x^{\prime}, y^{\prime}\right) ; \rho}\right| d x d y\right. \\
& \left.\quad+\iint_{D_{\rho / \gamma_{1}}\left(x^{\prime \prime}, y^{\prime \prime}\right) ; \rho}\left|t-t_{\left(x^{\prime \prime}, y^{\prime \prime}\right) ; \rho}\right| d x d y\right\} .
\end{aligned}
$$

We use the Hölder inequality to get

$$
\left|\bar{t}\left(x^{\prime}, y^{\prime}\right)-\bar{t}\left(x^{\prime \prime}, y^{\prime \prime}\right)\right| \leq C \rho^{\alpha},
$$

and, of course, similar inequalities for $\bar{r}, \bar{s}$. But, by combining (9) and (10),

$$
\iint_{D_{\rho / \gamma_{1}}\left(x_{0}, y_{0}\right)}\left\{\left|r-\bar{r}\left(x_{0}, y_{0}\right)\right|^{2}+\cdots+\left|t-\bar{t}\left(x_{0}, y_{0}\right)\right|^{2}\right\} d x d y \leq C \rho^{2+2 \alpha}
$$

for all $\left(x_{0}, y_{0}\right) \in \Omega$, and therefore $\bar{r}=r, \bar{s}=s, \bar{t}=t$ on the respective Lebesgue sets, which proves the theorem.

III. Proof of Theorem 2. In a neighborhood of $\left(x_{0}, y_{0}\right) \in \bar{\Omega}, \Omega$ is of the form

$$
\Omega_{\hat{R}}:=\Omega \cap D_{\hat{R}}=\left\{(x, y) \in D_{\hat{R}} \mid G(x, y)<0\right\}
$$

for some $\hat{R}, 0<\hat{R} \leq 1$, where $G(x, y) \in C^{2, \alpha}\left(\bar{D}_{\hat{R}}\right), G_{x}^{2} \geq 1 / \kappa>0$. We apply the variable transformation $\xi=G(x, y), \eta=y$, in order to flatten $\partial \Omega \cap D_{\hat{R}}$. We calculate the derivatives of $\hat{z}(\xi, \eta):=z(x(\xi, \eta), y(\xi, \eta))$,

$$
\begin{gathered}
\left(\begin{array}{l}
p \\
q
\end{array}\right)=\left(\begin{array}{ll}
G_{x} & 0 \\
G_{y} & 1
\end{array}\right)\left(\begin{array}{l}
\hat{p} \\
\hat{q}
\end{array}\right), \\
\left(\begin{array}{ll}
r & s \\
s & t
\end{array}\right)=\left(\begin{array}{ll}
G_{x} & 0 \\
G_{y} & 1
\end{array}\right)\left(\begin{array}{ll}
\hat{r} & \hat{s} \\
\hat{s} & \hat{t}
\end{array}\right)\left(\begin{array}{cc}
G_{x} & G_{y} \\
0 & 1
\end{array}\right)+\hat{p}\left(\begin{array}{ll}
G_{x x} & G_{x y} \\
G_{x y} & G_{y y}
\end{array}\right) .
\end{gathered}
$$

Hence

$$
\left(\begin{array}{cc}
r+C & s-B \\
s-B & t+A
\end{array}\right)=\left(\begin{array}{ll}
G_{x} & 0 \\
G_{y} & 1
\end{array}\right)\left(\begin{array}{ll}
\hat{r}+\hat{C} & \hat{s}-\hat{B} \\
\hat{s}-\hat{B} & \hat{t}+\hat{A}
\end{array}\right)\left(\begin{array}{cc}
G_{x} & G_{y} \\
0 & 1
\end{array}\right)
$$

where

$$
\begin{aligned}
& \left(\begin{array}{cc}
\hat{C} & -\hat{B} \\
-\hat{B} & \hat{A}
\end{array}\right) \\
& \quad:=\frac{1}{G_{x}^{2}}\left(\begin{array}{cc}
1 & 0 \\
-G_{y} & G_{x}
\end{array}\right)\left\{\left(\begin{array}{cc}
C & -B \\
-B & A
\end{array}\right)+\hat{p}\left(\begin{array}{ll}
G_{x x} & G_{x y} \\
G_{x y} & G_{y y}
\end{array}\right)\right\}\left(\begin{array}{cc}
1 & -G_{y} \\
0 & G_{x}
\end{array}\right) .
\end{aligned}
$$

Let

$$
\hat{\Delta}:=\Delta / G_{x}^{2} \geq 1 / \kappa c>0
$$

then $\hat{z}$ solves the elliptic Monge-Ampère equation

$$
(\hat{r}+\hat{C})(\hat{t}+\hat{A})-(\hat{s}-\hat{B})^{2}=\hat{\Delta} \text {. }
$$


When working near the boundary of $\Omega$, we can therefore assume w.l.o.g. that $\partial \Omega$ is flat.

From now on, we can follow the proof of Theorem 1, resp. [15], working in sets of the form $\Omega_{R}=\Omega \cap D_{R}$, resp. half-discs. We observe that the Legendre-like transformation preserves the flatness of $\partial \Omega \cap D_{\hat{R}}$ and introduce zero-boundary data of $y(u, v)$, resp. $\eta(u, v)$ as in [15]. As far as the Campanato technique is concerned, we can refer to Lemma 12 .II of [3, p. 355], for an inequality of the form (7) for halfdiscs, instead of referring to Lemma 8 .II of [3, p. 338], or to Giaquinta's book. Note that we have to use an inequality of the form (8) for the nontangential derivative, i.e., for $y_{u}$, resp. $\eta_{u}$. Theorem 2 is thus proved.

\section{REFERENCES}

1. A. D. Alexandrow, Die innere Geometrie der konvexen Flächen, Akademie-Verlag, Berlin, 1955.

2. I. Ya. Bakel'man, Generalized solutions of the Monge-Ampère equations, Dokl. Akad. Nauk SSSR 114 (1957), 1143-1145.

3. S. Campanato, Equazioni ellittiche del $\mathrm{II}^{\circ}$ ordine e spazi $\mathcal{L}^{(2, \lambda)}$, Ann. Mat. Pura Appl. (4) 69 (1965), 321-381.

4. __ Sistemi ellittici in forma divergenza. Regolarità all'interno, Quaderni, Scuola Normale Superiore Pisa, Pisa, 1980.

5. M. Giaquinta, Multiple integrals in the calculus of variations and nonlinear elliptic systems, Ann. of Math. Studies, no. 105, Princeton Univ. Press, Princeton, N.J., 1983.

6. E. Heinz, Interior estimates for solutions of elliptic Monge-Ampère equations, Partial Differential Equations, Proc. Sympos. Pure Math., vol. 4, Amer. Math. Soc., Providence, R. I., 1961, pp. 149-155.

7. H. Lewy, A priori limitations for solutions of Monge-Ampère equations, Trans. Amer. Math. Soc. 37 (1935), 417-434.

8. L. Nirenberg, On nonlinear elliptic partial differential equations and Hölder continuity, Comm. Pure Appl. Math. 6 (1953), 103-156.

9. A. V. Pogorelov, Monge-Ampère equations of elliptic type, Noordhoff, Groningen, 1964.

10. I. Kh. Sabitov, The regularity of convex regions with a metric that is regular in the Hölder classes, Siberian Math. J. 17 (1976), 681-687.

11. M. V. Safonov, On the classical solution of Bellman's elliptic equation, Soviet Math. Dokl. 30 (1984), 482-485.

12. F. Schulz, Über elliptische Monge-Ampèresche Differentialgleichungen mit einer Bemerkung zum Weylschen Einbettungsproblem, Nachr. Akad. Wiss. Göttingen II: Math. Phys. Kl. 1981, 93108.

13. $\_$, Über die Differentialgleichung $r t-s^{2}=f$ und das Weylsche Einbettungsproblem, Math. Z. 179 (1982), 1-10.

14. _ـ A priori estimates for solutions of Monge-Ampère equations, Arch. Rational Mech. Anal. 89 (1985), 123-133.

15. __ Boundary estimates for solutions of Monge-Ampère equations in the plane, Ann. Scuola Norm Sup. Pisa Cl. Sci. (4) 11 (1984), 431-440.

16. __ Über nichtlineare, konkave elliptische Differentialgleichungen, Math. Z. 191 (1986), 429448.

17. N. S. Trudinger, Regularity of solutions of fully nonlinear elliptic equations, Boll. Un. Mat. Ital. (6) A3 (1984), 421-430.

Department of Mathematics, The University of IOWA, IOWA City, IOWA 52242 